\title{
Integrative gene transfer in the truffle Tuber borchii by Agrobacterium tumefaciens-mediated transformation
}

\author{
Andrea Brenna', Barbara Montanini², Eleonora Muggiano ${ }^{1}$, Marco Proietto ${ }^{1}$, Patrizia Filetici ${ }^{3}$, Simone Ottonello $^{2}$ \\ and Paola Ballario ${ }^{1,3^{*}}$
}

\begin{abstract}
Agrobacterium tumefaciens-mediated transformation is a powerful tool for reverse genetics and functional genomic analysis in a wide variety of plants and fungi. Tuber spp. are ecologically important and gastronomically prized fungi ("truffles") with a cryptic life cycle, a subterranean habitat and a symbiotic, but also facultative saprophytic lifestyle. The genome of a representative member of this group of fungi has recently been sequenced. However, because of their poor genetic tractability, including transformation, truffles have so far eluded in-depth functional genomic investigations. Here we report that A. tumefaciens can infect Tuber borchii mycelia, thereby conveying its transfer DNA with the production of stably integrated transformants. We constructed two new binary plasmids (pABr1 and $\mathrm{pABr} 3$ ) and tested them as improved transformation vectors using the green fluorescent protein as reporter gene and hygromycin phosphotransferase as selection marker. Transformants were stable for at least 12 months of in vitro culture propagation and, as revealed by TAIL- PCR analysis, integration sites appear to be heterogeneous, with a preference for repeat element-containing genome sites.
\end{abstract}

Keywords: Tuber spp; Truffles; Agrobacterium tumefaciens-mediated transformation; T-DNA; binary plasmid; Green fluorescent protein; Hygromycin phosphotransferase; TAIL-PCR

\section{Introduction}

The ascomycete Tuber borchii is a hypogeous fungus (genus, Tuber; family Tuberaceae; order Pezizales) that establishes a beneficial mutualistic interaction ('ectomycorrhiza') with the roots of many tree species. T. borchii has also a pronounced saprobiotic capacity and can be grown in vitro (albeit quite slowly) as vegetative free-living mycelium in the absence of a plant host. This mixed symbiotic/saprophytic lifestyle (Hebe et al. 1999), together with the lack of asexual spores amenable to in vitro culture has hampered the genetic manipulation of this fungus and obscured our understanding of its complex life cycle. Tuber fruitbodies ('truffles') lack an active system for the dispersal of spores, which are disseminated by the action of mycophagous animals (Pegler et al.

\footnotetext{
* Correspondence: paola.ballario@uniroma1.it

'Pasteur Cenci Bolognetti Foundation, c/o Department of Biology and Biotechnology "Charles Darwin", Sapienza University, Piazzale A. Moro 5, 00185 Rome, Italy

${ }^{3}$ Institute of Biology and Molecular Pathology, CNR, 00185 Rome, Italy Full list of author information is available at the end of the article
}

1993). Another peculiarity of certain Tuber spp. is the high commercial value of their fruitbodies which are prized as gourmet food. Tuber melanosporum and T. borchii are the two most studied truffle species. Sequencing and annotation of the T. melanosporum genome, the first symbiotic Ascomycete to be sequenced, revealed many aspects of truffles' biology and genetic organization, including their massive content of repeated transposable elements and their heterotallic mode of conjugation and ability to outcross through the action of two distinct mating type loci (Martin et al. 2010). T. borchii is the truffle that can be more easily handled under laboratory conditions.

With the acquisition of new genome sequence data, functional genetic studies are all the more necessary to decipher gene function. Reverse genetics in Tuber has been hindered, however, by the absence of a wellestablished stable transformation system. An effective approach in this direction is represented by Agrobacterium tumefaciens-mediated (ATM) transformation of intact hyphae. This technique was first successfully applied to fungi 
19 years ago (Bundock et al. 1995) by exploiting the natural ability of $A$. tumefaciens to transfer a portion of its DNA to a foreign infected organism, most notably dicotyledonous plants. To date, ATM transformation is applied to the study of a variety of fungal species, including Ascomycetes, Basidiomycetes and Zygomycetes. The transfer DNA (T-DNA), located on a $>200 \mathrm{~kb}$ tumor-inducing (Ti) plasmid, is flanked by two 25 bp direct imperfect repeats, known as left border (LB) and right border (RB) sequences, which also include the vir genes encoding the genetic factors required for transfer (Zupan et al. 2000). Recent improvements of ATM transformation include the optimization of temperature and co-cultivation conditions, and the development of new selection markers (Michielse et al. 2005). We previously described an ATM transformation procedure for $T$. borchii (Grimaldi et al. 2005), which due to the insertion of transgenic DNA driven by the T-DNA, and consequent lack of stability, could not be effectively exploited for functional genomics studies. Building upon these earlier attempts, in the present work we improved transformation conditions through the development of two new vectors and also gained insight into the fate of the transferred T-DNA, which was found to be randomly integrated, with a preference for repeat element-containing genome sites. These results represent an important technical advancement for the molecular biological investigation of truffles, which will be instrumental to the construction of mutant strain collections.

\section{Materials and methods}

\section{Strains and media}

T. borchii Vittad. mycelia (isolate ATCC 95640) were grown and propagated in the dark at $24^{\circ} \mathrm{C}$ on potatodextrose medium with agar $39 \mathrm{~g} / \mathrm{l}$ (PDA: $0.2 \%$ peptone, $0.2 \%$ yeast extract, $1.8 \%$ glucose, $0.5 \%$ potato starch, 1.5\% agar; Liofilchem), as described (Ambra et al. 2004). For easy medium replacement, mycelial cultures were usually grown on sterile dialysis membranes. For liquid culture replacement, mycelial cultures were inoculated in potato-dextrose liquid medium (PDB, without agar). A. tumefaciens was grown and propagated in LuriaBertani (LB) (1\% tryptone, $0.5 \%$ yeast extract, $1.5 \%$ agar) solid or liquid (w/o agar) medium at $28^{\circ} \mathrm{C}$ in the presence of appropriate marker selection supplements. After co-cultivation, mycelia were transferred onto Evans minimal medium (1x Vogel's salts, 1\% glucose, $2 \mathrm{mg} / \mathrm{l}$ vitamin B1, 1.5\% agar). The hypervirulent Agrobacterium strain AGL-1 [(C58 pTiBo542) recA::bla, T-region deleted Mop (+) Cb (R)] was kindly provided by Peter Romaine (Department of Plant Pathology, Pennsylvania State University, University Park, PA, USA) (Lazo et al. 1991). Carbenicillin (Sigma-Aldrich, St Louis, MO) $100 \mu \mathrm{g} / \mathrm{ml}$ was used for AGL-1 propagation. The GV3101 strain (background C58; Ti-plasmid cured) was kindly provided by
Paolo Costantino (Department of Biology and Biotechnology "La Sapienza" University, Rome, Italy). Rifampicin $(25 \mu \mathrm{g} / \mathrm{ml})$ and gentamicin $(25 \mu \mathrm{g} / \mathrm{ml})$, both provided by Duchefa Biochemie, were used for GV3101 propagation.

\section{Plasmids}

The binary plasmid vector pBGgHg (Chen et al. 2000) consists of a pCAMBIA 1300 (CAMBIA, Canberra, Australia), backbone containing the Escherichia coli hygromycin B phosphotransferase $(h p h)$ gene and the enhanced green fluorescent proteins (egfp) gene, both under control of the strong and constitutive glyceraldehyde-3- phosphate dehydrogenase $(g p d)$ promoter from Agaricus bisporus and the CaMV35S terminator. The plasmid was kindly provided by Peter Romaine (Department of Plant Pathology, Pennsylvania State University). The binary vector pABr1 (Figure 1a) was derived from pCAMBIA 1302 (CAMBIA, Canberra, Australia) through the incorporation of a region extracted from pCT74 (Ciuffetti et al. 1997; Lorang et al. 2001). This region contains a synthetic green fluorescent protein $(s g f p)$ gene under control of the strong and constitutive ToxA promoter from Pyrenophora tritici-repentis and a hygromycin B phosphotransferase (hph) gene under control of the constitutive promoter $p d g p$ from Agaricus bisporus. To generate $\mathrm{pABr} 1$, both starting plasmids were subjected to EcoRI-XhoI digestion, which removed the TDNA region of PCAMBIA 1302 containing the plant $h p h$ gene under control of the CaMV35S promoter, while the GFP gene controlled by the CaMV35S promoter was retained. The binary plasmid vector $\mathrm{pABr} 3$ is a shortened version of $\mathrm{pABr} 1$ from which the region containing the GFP gene under control of the CaMV35S promoter used for plant transformation was deleted.

\section{ATM transformation}

T. borchii mycelia were pre-grown in the dark at $24^{\circ} \mathrm{C}$ for 7 days on cellophane-overlaid PDA plates and then transferred to PDA medium containing $200 \mu \mathrm{M}$ acetosyringone (AS) to stimulate Agrobacterium virulence (outlined in Additional file 1: Figure S1). Three days before the start of co-cultivation, pre-transformed Agrobacterium cells were grown at $28^{\circ} \mathrm{C}$ for 2 days in the presence of the appropriate selection marker antibiotics $(100 \mu \mathrm{g} / \mathrm{ml}$ carbenicillin for AGL-1; $25 \mu \mathrm{g} / \mathrm{ml}$ each of rifampicin and gentamycin for GV3101; $30 \mu \mathrm{g} / \mathrm{ml}$ kanamycin for both vectors). On the day of co-cultivation, a fresh overnight bacterial culture with an optical density at $600 \mathrm{~nm}\left(\mathrm{OD}_{600 \mathrm{~nm}}\right)$ of 1.2-1.5 was diluted to an $\mathrm{OD}_{600 \mathrm{~nm}}$ of 0.075 with antibiotic-supplemented medium also containing $200 \mu \mathrm{M}$ AS and grown for $4 \mathrm{~h}$ to an $\mathrm{OD}_{600 \mathrm{~nm}}$ of 0.3 . After reaching this cell density, $50 \mu \mathrm{l}$ of bacteria in LB $\left(1.5 \times 10^{3}\right.$ cells $\left./ \mu \mathrm{l}\right)$ were overlaid onto T. borchii mycelia and co-cultivation was continued for 3 days at $22-24^{\circ} \mathrm{C}$. Following co-cultivation, mycelia 


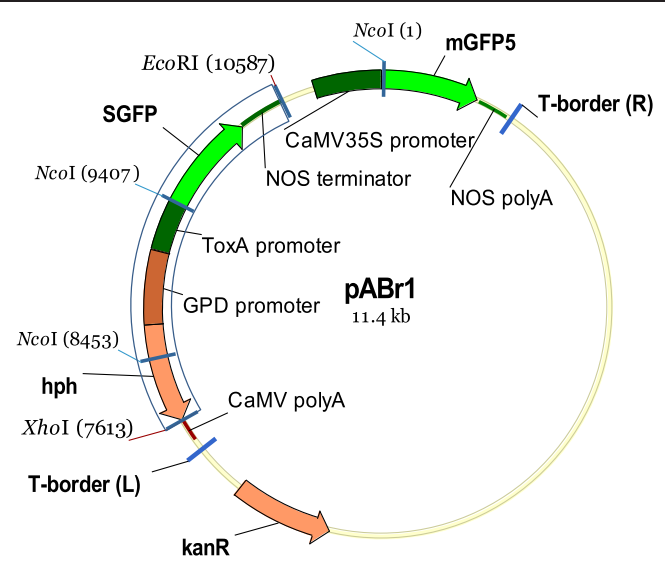

b
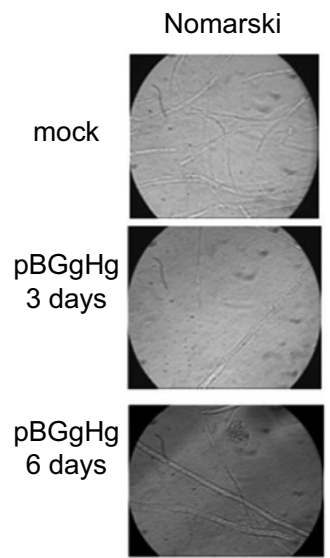

$\mathrm{pABr} 1$

3 days

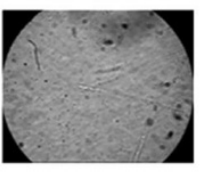

$\mathrm{pABr} 1$

6 days

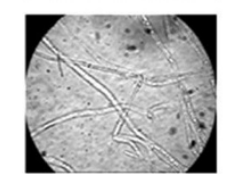

GFP
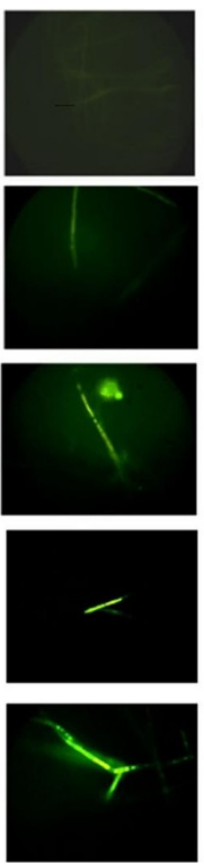

C

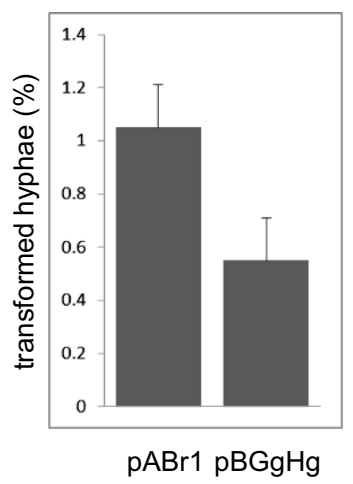

d

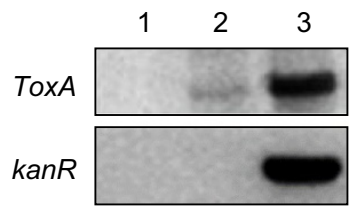

Figure 1 ATM-transformation with the pABr1 vector. a) $\mathrm{pABr} 1$ (11.4 kb) vector map: Hygromycin (R), hygromycin phosphotransferase gene (hph); Kanamicin resistance gene (kanR); ToxA promoter, promoter sequence from P. tritici-repentis; SGFP, the S65T variant of the green fluorescent protein (GFP); CAMV35S cauliflower mosaic virus promoter; $m$ GFP5, folding-enhanced green fluorescent protein variant; NOS polyA, Nopaline synthase terminator sequence; T-border (R) and T-border (L), right (RB) and left (LB) borders of A. tumefaciens T-DNA. The blue highlighted box is the plasmid region derived from $\mathrm{pCT74}$. b) Images (100x magnification) of $T$. borchii hyphae transformed with either the pABr1 or the pBGgHg vector, plus an untransformed control (mock), obtained by phase-contrast (Nomarski) and GFP fluorescence microscopy (GFP). The type of treatment, vector and co-cultivation times are indicated on the left; a merge of the Nomarski and GFP images is shown in the rightmost panels. c) Quantification of transformed hyphae obtained with $\mathrm{pBGgHg}$ or $\mathrm{pABr}-1$, expressed as percentage with respect to the total number of hyphae ( 4500) present in each analyzed section. Data are the mean \pm s.e.m. of at least five independent experiments. d) PCR amplification with ToxA-specific primers of total DNA extracted from mock-infected mycelia (lane 1), pABr1-transformed mycelia (lane 2), and pABr1/AGL-1 bacterial cells (lane 3) is shown in the upper panel. The results of PCR amplification of the same samples with kanR-specific primers are shown in the lower panel.

were washed with sterile $d_{d H_{2}} \mathrm{O}$ and $400 \mu \mathrm{M}$ cefotaxime, and mycelial samples were sliced and examined by confocal microscopy. The remaining mycelia were transferred to new plates of Evans minimal medium containing $400 \mu \mathrm{M}$ cefotaxime (to inhibit bacterial growth) and
$15 \mu \mathrm{g} / \mathrm{ml}$ hygromycin (to aid in transformant hyphae isolation). Additional mycelial samples were analyzed after 6 days of co-cultivation, and the remaining mycelia were monitored over time for hygromycin B resistance and GFP expression. 


\section{Fluorescence and confocal microscopy}

Sections $(3 \times 3 \mathrm{~mm})$ of either control, untransformed T. borchii mycelia or mycelia co-cultivated with $A$. tumefaciens for 3 days were washed 5 times with distilled water and cefotaxime $(400 \mu \mathrm{M})$ prior to microscopic analysis. An Axioskop 2 microscope (Carl Zeiss International, Oberkochen, Germany) equipped with a 100X oil immersion objective (Plan Neofluar), a Zeiss filter set (green excitation filter 450-490 HB) and a Zeiss AttoArc2 HB-100 W mercury lamp were used for fluorescence microscopy analysis. Images were captured with a Microcolor camera (RGB-MS-C; CRI, Boston, MA) and processed with Diffraction Micro CCD software and Adobe Photoshop (Adobe System, San Jose, CA). Typical exposure times were $30-60 \mathrm{~ms}$ for the SGFP and Nomarski images. Confocal laser scanning microscopy was performed with a Leica microscope equipped with a TCS SP2 Laser ( $\mathrm{Ar} / \mathrm{Kr}$, Gre/Ne, He/Ne; standard phase contrast plus Normaski contrast). SGFP was excited with a $488-\mathrm{nm}$ laser line and detected at 515-530 nm.

\section{DNA extraction and PCR analysis}

Mycelia to be analyzed by confocal microscopy were washed 5 times with $\mathrm{ddH}_{2} \mathrm{O}$ and $400 \mu \mathrm{M}$ cefotaxime and then disrupted by freezing in liquid nitrogen. DNA was extracted from frozen mycelia using the DNeasy Plant Minikit (QIAGEN N. V., Hilden, Germany) as per manufacturer's instructions. The amplification protocols described in (Grimaldi et al. 2005) were employed for PCR analysis of the aminoglycoside 3'-phosphotransferase $(k a n R)$ and $s g f p$ genes, using the oligonucleotide primers listed below.

kanR primers (to amplify a region comprised within the binary plasmid vector):

kanR_Fw: 5'-GGTCATGCATTCTAGGTACT-3'; kanR_ Rw: 5'-AATGGCTAAAATGAGAATAT-3'.

$S g f p$ primers (to amplify a region comprised within the T-DNA):

sgfp_Fw: 5' - CACATGAAGCAGCACGACTT-3'; sgfp_ Rw: 5'-TGCTCAGGTAGTGGTTGTCG-3';

ToxA promoter region (also comprised within the T-DNA):

ToxA_Fw: 5' -GGGGAATTCTAGTGGAACTGATTG GAATGCA-3';

ToxA_Rw: 5' -GGGGATAGAACCCATGGCCTATAT-3'

\section{DNA blot analysis}

Genomic DNA from transformed and untransformed (negative control) T. borchii mycelia $(10 \mu \mathrm{g}$ each) plus the same amount of pABr1 plasmid DNA (positive control) were digested with NcoI. A separate sample of pABr1 was digested in parallel in order to generate a pair of DNA fragments to be used as probes. Four fragments were obtained from NcoI-XhoI digestion, including an $840 \mathrm{bp}$ fragment that was used as probe. This fragment comprises a region (located around the T-DNA junction site) that encompasses the site of recombination with genomic DNA. The second probe was obtained by NcoI digestion, which produced three different fragments. The fragment that was utilized as probe was $954 \mathrm{bp}$ in length (from the middle of the hygromycin phosphotransferase cassette to the ToxA promoter). This probe matches the region of the T-DNA that is transferred to $T$. borchii hyphae. Radioactive labeling with approximately $20 \mathrm{ng}$ of purified templates, blotting, hybridization and washing were performed as described (Green and Sambrook 2012; Montanini et al. 2003).

\section{TAIL-PCR amplification, cloning and sequencing of T-DNA integration sites}

Genomic DNA sequences flanking the T-DNA were amplified by Thermal Asymmetric Interlaced PCR (TAIL-PCR) as described in (Wang and Li 2008). The following oligonucleotides were used as primers to amplify the Left Border (LB) and the Right Border (RB) regions, in combination with the degenerate, genome-sequence targeting primer AD1.

LB1 5'-GGGTTCCTATAGGGTTTCGCTCATG-3' LB2 5'-CATGTGTTGAGCATATAAGAAACCCT-3' LB3 5' -GA ATTAATTCGGCGTTAATTCAGT-3'

RB1 5' -GGCATGGCCGTCGTTTTACAAC-3' RB2 5' AACGTCGTGACTGGGAAAACCCT-3' RB3 5'-CCCT TCCCAACAGTTGCGCA-3'

\section{AD1 5' -WGTGNAGWANCANAGA-3'}

DNA amplified by TAIL-PCR was cloned into the pDrive A/T vector (QIAGEN) according to the manufacturer's instructions, followed by DNA sequence analysis of plasmids isolated from transformant colonies.

\section{Other procedures}

Mycelial sections $(3 \times 3 \mathrm{~mm})$ containing 4500 hyphal apexes, as estimated by counting with a Burker chamber, were used for transformation efficiency determinations. The fraction (\%) of transformed hyphae was obtained by dividing the number of fluorescent hyphae (average of multiple determinations) by 4500 ; data were calculated as the mean \pm standard error of the mean (s.e.m.) of at least five independent replicates.

The Vector NTI ${ }^{\circ}$ Software (Life Technologies) was used for drawing plasmid maps. BLAST searches were conducted against the $T$. melanosporum genome sequence at the MycorWeb site (http://mycor.nancy.inra.fr).

\section{Results}

ATM transformation of $T$. borchii mycelia using a Pyrenophora ToxA promoter-bearing binary vector We previously documented the production of transformed, GFP-expressing $T$. borchii mycelia with the use 
of ATM and the pBGgHg plasmid (Grimaldi et al. 2005). However, transformation efficiency was rather low (0.2$0.5 \%$ ) with a fairly weak GFP signal that was lost after a few weeks, possibly due to the lack of stable integration. In order to improve transformation efficiency and to achieve higher GFP expression levels as well as stable transformants, a new series of cloning vectors for ATM transformation was constructed. We first created a binary vector, named pABr1 (Figure 1a), with the sgfp gene under control of the ToxA promoter from the ascomycete $P$. tritici-repentis (Ciuffetti et al. 1997), inserted within the T-DNA region, which also contains the hygromycin phosphotransferase $(h p h)$ gene utilized for positive selection of transformants. The sgfp gene, which codes for the S65T variant of GFP, is a commonly utilized transformation marker in fungi (Niwa 2003) and use of the ToxA promoter has previously been shown to enhance the expression of reporter genes in various fungi including Neurospora crassa (Lorang et al. 2001). Our goal was to couple the enhanced strength of the ToxA promoter with the increased stability of SGFP in order to obtain a stronger fluorescence signal and thus an improved visualization of transformed hyphae. To this end, we performed ATM transformation by treating mycelia with the AGL-1 strain, carrying either PABr1 or $\mathrm{pBGgHg}$, and comparing the transformation efficiency of these two vectors. After 3 days of co-cultivation (outlined in Additional file 1: Figure S1), a subset of mycelia was transferred to new antibiotic-containing plates (ampicillin, cefotaxime and hygromycin B) and samples were taken for hyphal section visualization by confocal microscopy. This allowed the identification of fluorescent, GFP-producing hyphae, by comparison with the background signal associated with control mycelia transformed with the empty AGL-1 strain (Figure 1b). The diffuse fluorescence background present in mock-transformed samples, contrasted with the localized signal observed in the apical hyphae of T. borchii transformed with AGL-1 carrying the binary vector. Transformation efficiency obtained with the pABr1 vector after 3 days of cocultivation was consistently found to be at least twofold higher $(\geq 1 \%)$ than that obtained with $\mathrm{pBGgHg}$ using the same AGL-1 A. tumefaciens strain (Figure 1c). This was calculated as the ratio between fluorescent hyphae and the total number of hyphae per section ( 4500). When the analysis was repeated at 6 days, i.e. after 3 additional days since the end of co-cultivation, the hyphae appeared more fluorescent, likely due to SGFP accumulation (Figure 1b), but without any appreciable change in the relative number of fluorescent hyphae (data not shown). Still, the SGFP signal in pABr1-transformed hyphae appeared stronger than that of hyphae transformed with the $\mathrm{pBGgHg}$ vector, thus facilitating the identification of transformants grown over time. Transformation was also verified by PCR amplification of the ToxA promoter using genomic-DNA extracted from transformed mycelia as template (Figure 1d). To confirm that ToxA amplification did indeed result from the mobilized T-DNA rather than from residual bacteria, we performed a parallel PCR analysis using a pair of primers annealing to the kanamycin resistance $(\mathrm{kan} R)$ gene region that is present in the plasmid outside of the T-DNA. The absence of a kanR amplification signal ruled out a false positive result caused by residual contaminating bacteria. We then used a different Agrobacterium strain (GV3101) to verify that the improved transformation efficiency obtained with pABr1 was a genuine effect of the plasmid vector, rather than an indirect effect of the specific $A$. tumefaciens strain utilized for transformation. The results obtained with GV3101 confirmed that also in this strain the transformation efficiency yielded by pABr1 was at least two-fold higher than that obtained with pBGgHg (Additional file 1: Figure S2a,b). However, global efficiency with GV3101-mediated transformation was generally lower than that obtained with AGL-1 (Additional file 1: Figure S2c), suggesting that not all A. tumefaciens strains are equally effective for Tuber transformation. Also in this case, the data were confirmed by PCR analysis, which revealed an $s g f p$ amplicon in the case of DNA extracted from transformed mycelia, but not in the case of mock-infected mycelia (Additional file 1: Figure S2d). Furthermore, a stronger GFP signal was systematically observed in pABr1 transformants compared to transformants obtained with the $\mathrm{pBGgHg}$ vector utilized in previous studies (Grimaldi et al. 2005). Altogether the data confirm the superior performance of the newly designed pABr1 vector.

\section{T-DNA transformation occurs by random integration in the Tuber genome}

As described previously (Bundock and Hooykaas 1996), the T-DNA can either be integrated into the host genome or transferred and incorporated as stable, extrachromosomal T-DNA elements that can form circular structures in plants (Singer et al. 2012). To investigate this point, we used a phenotype-based approach. We hypothesized that if the T-DNA was integrated in the Tuber genome, the same transformant section analyzed at different times after transformation should show a progressive increase in the fluorescence of neighboring hyphae, resulting from multiple nuclear divisions of hyphae containing the integrated transgene. We thus isolated regions of AGL-1/pABr1-transformed mycelia containing one or very few transformed hyphae (visualized by confocal microscopy after 6 days of co-cultivation; see Figure $2 \mathrm{a}$ "6d") and, after sterile washing, put them back to liquid medium for an additional 15 days. Subsequent analysis by confocal microscopy revealed a four-fold increase in the 
b

\section{a}

Nomarski

GFP

$6 \mathrm{~d}$

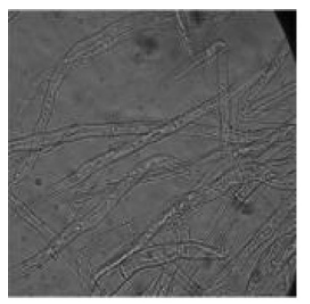

$21 \mathrm{~d}$
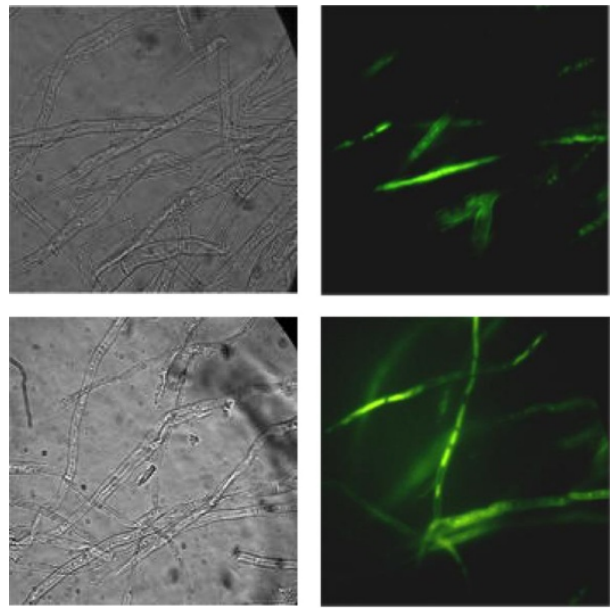

C

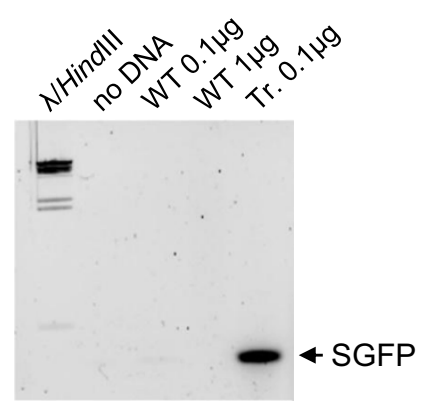

e

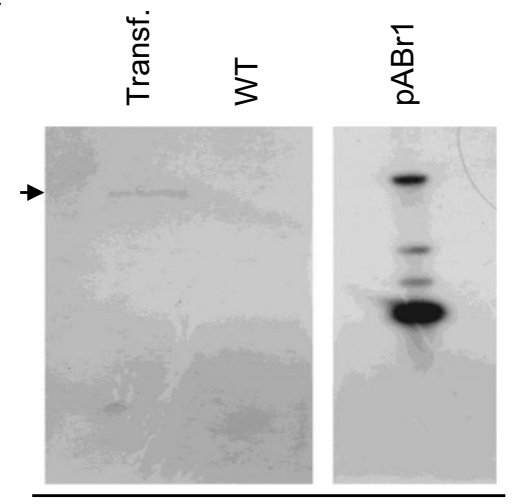

P1 probe

d

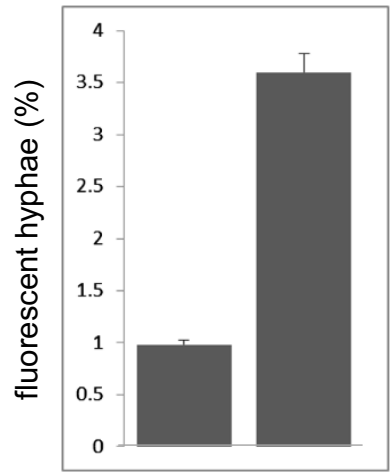

$6 d \quad 21 d$
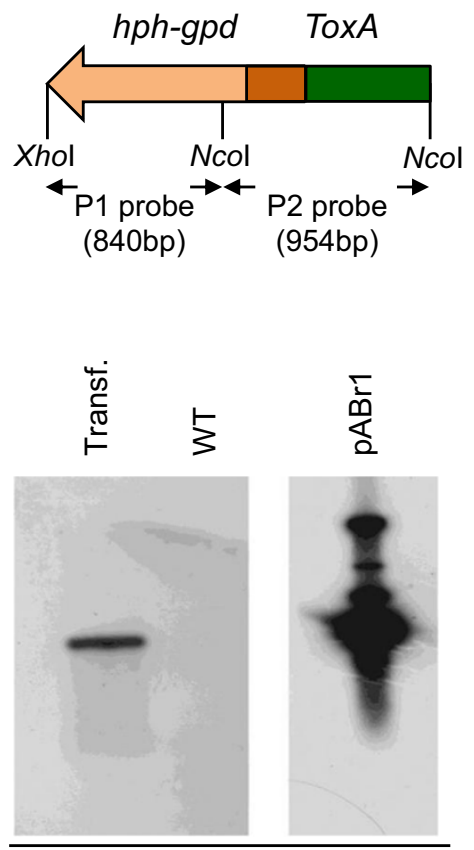

P2 probe

Figure 2 Following the fate of the T-DNA in pABr1-transformed hyphae. a) Sections $(3 \times 3 \mathrm{~mm})$ of transformed T. borchii mycelia analyzed by confocal microscopy (GFP wavelength) at the indicated times after Agrobacterium infection. b) Histogram representation of cumulative data produced by the experiments in panel a) expressed as percentage of fluorescent hyphae with respect to the total number of hyphae present in each section ( 4500). Data are the mean \pm s.e.m. of at least five independent experiments. c) PCR amplification with sgfp specific-primers of genomic DNA obtained from mock-transformed mycelia (WT) using $0.1 \mu \mathrm{g}$ (lane 3) and $1 \mu \mathrm{g}$ (lane 4) of template DNA, and from AGL-1/pABr1-transformed mycelia (0.1 $\mu \mathrm{g}$ of template DNA; lane5). A PCR negative control (no DNA) and size markers (NHIII) are shown in the two leftmost lanes. d) Schematic representation of the probes (P1 and P2) utilized for DNA blot analysis. e) DNA blot analysis of DNA extracted from AGL-1/pABr1-transformed mycelia ("transformant", lane 1), mock-transformed mycelia (WT, lane 2), and AGL-1/ABr-1 bacterial cells ( $p A B r 1$, lane 3), hybridized with the P1 probe, which detects T-DNA integration regions. After stripping, the same membrane was hybridized with the P2 control probe. 
number of contiguous fluorescent hyphae (see Figure 2a " $21 d$ " and b), in keeping with the hypothesis that transformation mainly occurs via stable integration rather than through the incorporation of extra-chromosomal elements. Transformation was confirmed at the molecular level by the detection of an sgfp PCR amplification product in transformed mycelia and the lack of a similar product when using increasing amounts of genomic DNA from mock infected wild-type mycelia as template (Figure 2c). To demonstrate the presence of integrated transgene copies, we further investigated the fate of the transgene by DNA blot analysis. Since very little amounts of genomic DNA can usually be obtained from Tuber mycelia, and the number of transformed nuclei is far less than that of untransformed nuclei, unique regions of integration were difficult to visualize. To overcome this problem, also considering that random, non-sequence specific T-DNA integration has been shown to be the main outcome of ATM-transformation in other fungi (Bundock et al. 2002), we mapped T- DNA insertions at the genomic level by hybridization analysis conducted with two specific probes (named P1 and P2).

The P1 probe was obtained by using an 840 bp sequence derived from NcoI-XhoI digestion of $\mathrm{pABr} 1$ as template (Figure $2 \mathrm{~d}$ ). P1 recognizes the T-DNA region between the left border and the $h p h$ sequence. This region presumably recombines with the Tuber genome as described in other fungi (Thomas and Jones 2007). The other probe (P2) was generated by using as template, a 954 bp region of the T-DNA obtained by NcoI digestion (Figure 2d). This probe recognizes an internal sequence comprising part of the $h p h$ gene, the $p d g p$ promoter and the entire ToxA promoter sequence, that is present regardless of the integrated or extra-chromosomal state of the T-DNA. The P2 probe was thus used as a positive control for hybridization experiments, to verify that DNA blotting had been performed correctly. Total genomic DNA from transformed and untransformed mycelia was then subjected to NcoI digestion, which would generate a smear of fragments recognized by the P1 probe only in the case of T-DNA integration. As shown in Figure 2e (left panel), a weak, P1 probe-positive band was only observed in transformed mycelia. The fact that the size of this band ( 10,000 bp) was much higher than expected (840 bp) strongly suggests the occurrence of an integration event. In the same sample, a stronger signal resulting from the sum of integrated and extra chromosomal T-DNA fragments of the same size, was observed with the P2-probe, which hybridized with a DNA fragment, located within the T-DNA region, having an expected size of $954 \mathrm{bp}$ (Figure 2e, right panel). Also in this case, the P2-associated signal was only observed in the case of DNA derived from transformed, but not mock-infected, mycelia. Altogether these data suggest the integration of at least some copies of the pABr1derived T-DNA in the Tuber genome.

\section{Improved transformation efficiency of the shortened pABr3 vector}

pABr1 is a binary vector containing the T-DNA sequence interposed between the left border and the right border. It also contains an unmodified $g f p$ gene, under control of the CaMV35S promoter and a NOS terminator (necessary for plant transformation), between the HindIII site and the right border of the T-DNA (Figure 1a). While contributing to, and considerably expanding the size of the T-DNA, this region is presumably not necessary for Tuber transformation and it was thus eliminated. The T-DNA of the resulting vector, designated as pABr3, only contained sequences derived from the pCT74 plasmid (hph gene, gpd promoter, ToxA promoter and the $s g f p$ reporter gene). We hypothesized that the smaller size of the T-DNA sequence harbored by pABr3, corresponding to approximately $1 / 3$ of the sequence contained in $\mathrm{pABr} 1$, may result in a higher transformation efficiency, thus increasing the number of transferred T-DNA copies. Indeed, as shown in Figure 3, a comparison of pABr1- and pABr3-transformed hyphae (obtained after 3 days of co-cultivation with the AGL-1/ pABr1 and AGL-1/pABr3 Agrobacterium strains) revealed an approximately 6-fold higher transformation efficiency with the latter binary vector, likely resulting from the combined effect of a smaller sized T-DNA and an enhanced expression of the SGFP reporter. Moreover, as revealed by confocal microscopy, the SGFP signal was found to be strikingly stable and diffuse even 1 year after the initial transformation, further indicating the increased performance of this optimized-transformation procedure.

Since it was previously impossible to obtain portions of Tuber mycelia capable of growing on media containing more than $15 \mu \mathrm{g} / \mathrm{ml}$ hygromycin B, we also analyzed the ability of AGL-1/pABr3-transformed mycelia to grow in the presence of increasing amounts of hygromycin B. To this end, transformed mycelia were transferred to plates containing $15 \mu \mathrm{g} / \mathrm{ml}$ hygromycin B and the outline of the mycelium was marked so to allow an easy visualization of mycelial clumps overgrowth over time. After 60 days (during which mycelia were transferred to fresh antibiotic-containing medium every 10 days), fullbodied mycelial clumps were found to propagate on medium containing $15 \mu \mathrm{g} / \mathrm{ml}$ of hygromycin B (Figure 3c, top panel). Following isolation and transfer of hygromycinresistant mycelial slices to media containing increasing concentrations of hygromycin $\mathrm{B}$, we found that transformants were able to propagate in the presence of hygromycin B concentrations as high as $300 \mu \mathrm{g} / \mathrm{ml}$ (Figure 3c, bottom panel). Mycelia subjected to this high-stringency 


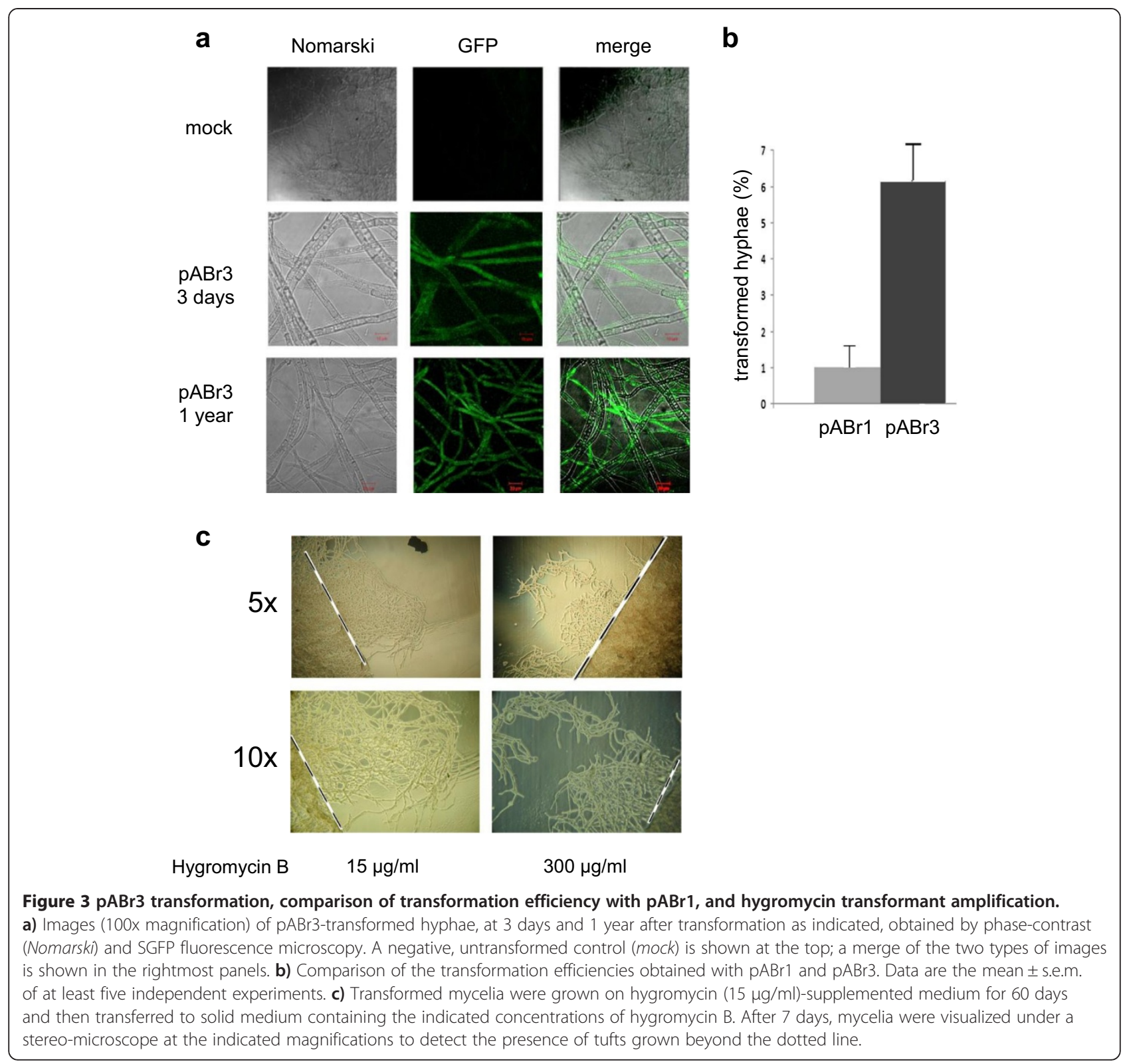

selection procedure were used as starting material for further molecular analyses (see below).

\section{Integration site identification by TAIL-PCR}

The above results suggest that $A$. tumefaciens transforms $T$. borchii via genomic integration of the T-DNA. We further investigated this point by isolating T-DNA integration sites comprised of unknown Tuber genome sequences flanking known sequences of the T-DNA borders. To overcome potential difficulties deriving from the syncytium organization of Tuber hyphae, which upon transformation become a mosaic with only one transformed nucleus surrounded by a multitude of untransformed nuclei, we used TAIL-PCR as a sensitive tool to recover flanking-DNA sequences (Liu and Whittier 1995).
This consisted of three subsequent PCR cycles amplifying a region interposed between the T-DNA border and the flanking genomic DNA using different, LB or RB, sitespecific primers and a degenerate primer. The high specificity of this reaction makes TAIL-PCR a powerful tool to verify T-DNA integration in fungal cells after ATM transformation (Rolland et al. 2003). DNA obtained from mycelia transformed with AGL-1/pABr3 and subjected to high-stringency hygromycin selection, together with DNA from control untransformed mycelia was used for TAILPCR analysis. While the first cycle of TAIL-PCR, conducted on the left border, generated non-specific amplification products, the last cycle yielded specific products that were only detectable in genomic DNA from transformed mycelia (Figure 4a). The same approach applied to 


\section{a}

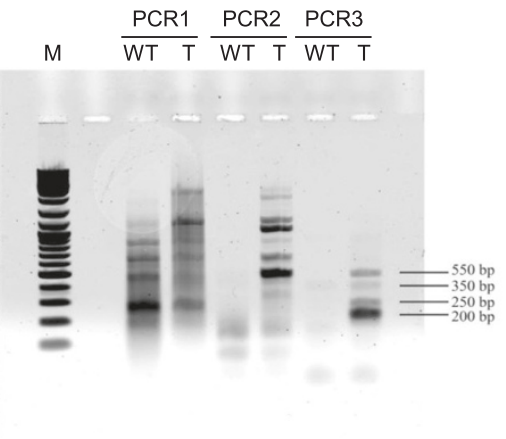

b

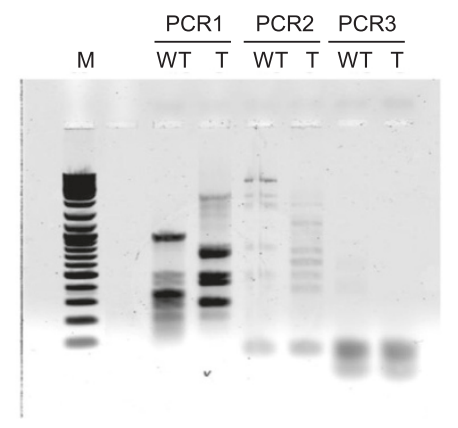

C

$>$ Clone 2 ( 250bp)
GTGATTAGTGCAGAACCAGAGAGAACCCCGCCAAGCAGCTCATATCGCAA ACGAGCTATCCATTCTCCTTACATCTACCAAAACCCACGATCCTATTATA TAAAAACATCCACCTGATCAATTAGTCAGTACAGTAGGGCACAGTCCACG ATTAACTTAATAACACATTGCGGACGTTTT TAATGTACTGAATTAACGCC GAATTAATTC

$>$ Clone 8 ( 250bp)

GTGATTAGTGCAGAACCACAGAGAACCCCGCCAAGCAGCTCATATCGCA ACGAGCTATCCATTCTCTTTACATCTACCAAAACCCACGATCCTATTAT TAAAAACATTCACCTGATCAATTAGTCAGTACAGTAGGGCACAGTCCACG ATTAACTTAATAACACATTGCGGACGTTTTTAATGTACTGAATTAACGCC GAATTAATTC

$>$ Clone $10 \quad(\sim 200 \mathrm{bp})$

AgATTTGTGGAGTAGCAGAGATAATAGTAGATTCCGTTACAGCCTCCTT

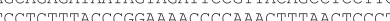
TAGTGCCCTCTGACACATGCAGCTCCCGGAATCAC
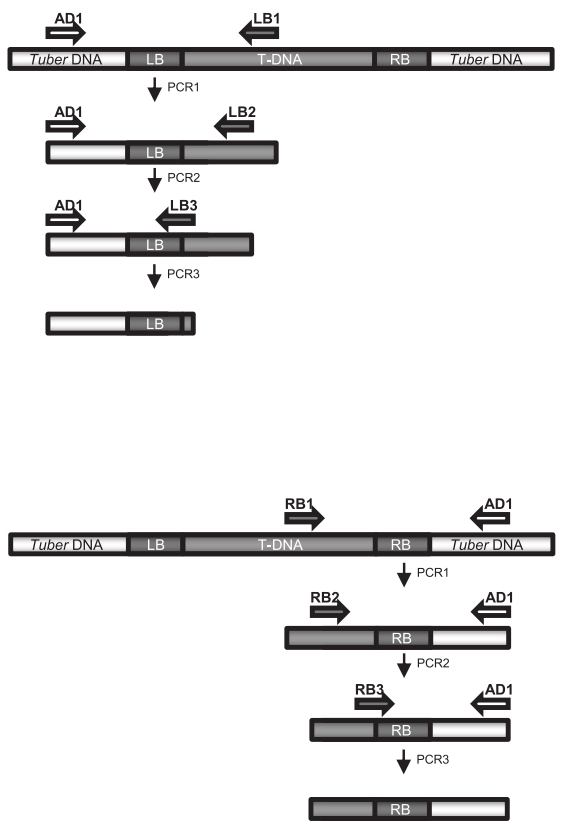

Clone 11 ( $550 \mathrm{bp})$

GTGATTAGTGGAGAAGCAGAGAGGCGGGAGGCGACAATCCTCTCGAGGAT CTTGGAGACCGTTTCCAGAAGGACGATAATTCGAAAAGAGGCCGGCGAGT CATAGGAGGGCTTCCCAGGCTTGTCGAGAACTACGCCGTGCGCTCGTTTT AGAGAGGCGGGGTGGTGGCCGTATACCAGGAGCGGTGAAAGAAGGGTGGT CAGAATGTGGGGGCAGGCTTTATGCAGGGTCTTTCAAACAGAGTAGGGAA TAGTATCCGGACCAGGGGCGGACGACGGAGAGCATTTTGAGAGGGCAGC GCTATCTCGCTCTGTTTCCTGCTCATTCTCATACTTCATACTTATCACC GTACCTACCACTCTCCAGCCTGTACCTATATATTGTGGTGTAAACAAATT GACGCTTAGACAACTTAATAACACATTGCGGACGTTTTTAATGTACTGA TTAACGCCGAATTAATTC

$>$ Clone $12 \quad(\sim 350 \mathrm{bp})$

AGATAGTGGAGAAGCAGAGAGAAGAGGAAAACCCCACCGGCCCTACCAC CTCAAGAGGAAGAACAGGACACCCTTACAGTCACAAATACTCCCAACCAT GTAGGCTCATCTGGGACTGCCAACAGAGCCCTTGAAGCCTACATTACAGT CAATGGCCACAAAGCGAAGCCCT MATHCGATCAGCAACTATAGAAATA ACCTAATCTCTGGAAAATTCATCTCCACCTTCCT TAATGTACTGAATTAA

Figure 4 TAIL-PCR and integration site identification. a) TAlL-PCR amplification of DNA fragments covering pABr3 integration sites. Representative results obtained from three amplification runs (PCR1, PCR2, PCR3) carried out with three nested primers annealing with the left border (LB) of the T-DNA (LB1, LB2 and LB3) and a degenerate primer annealing with the neighboring genome sequence (AD1) (see explanatory scheme on the right and "Materials and Methods" for details). Template genomic DNA was extracted from a pABr3-transformed mycelium (T) and from a mock-transformed mycelium (WT); size markers (M) are in the leftmost lane. b) Amplification of the right border (RB). c) Integration site sequences retrieved from TAlL-PCR analysis of transformants \#2, \#8, \#10, \#11 and \#12; The underlined and highlighted in bold sequences represent the portion of the T-DNA adjacent to the unknown sequence obtained by PCR.

the right border did not yield any amplicon (Figure 4b). This is a common problem previously described in various plant studies (Mazars et al. 1991) and it is likely caused by illegitimate recombination events that also occur during fungal transformation (Choi et al. 2007). Sequence analysis of transformant-specific LB amplification products revealed T-DNA/genomic DNA junction sites (Figure 4c). The non-T-DNA portions of the sequences thus retrieved were used as queries for a BLAST search performed against the heterologous, but likely closely related, $T$. melanosporum genome (Martin et al. 2010). Two sequences (\#10 and \# 11) yielded positive matches, corresponding, respectively, to a region between two closely located transposable elements and to a retrotransposable element gene. The apparent lack of positive matches for the other non-T-DNA sequences retrieved from TAIL-PCR may be explained by the relatively short length of such sequences compared to the extremely high number and variability of transposons and other repeated elements in the T. melanosporum genome utilized as reference for BLAST search. TAIL-PCR analysis thus provided final proof of the integrative transfer of the T-DNA region into the Tuber genome.

\section{Discussion}

Tuber spp. are ecologically and economically important filamentous Ascomycetes. These fungi, whose fruitbodies 
are commonly known as truffles (Pegler et al. 1993), have a subterranean habitat and establish a symbiotic relationship with several trees and shrubs via ectomycorrhiza formation (Hebe et al. 1999). Crucial for the success of the ectomycorrhizal interaction is the mutualistic exchange of nutrients between the symbiont and the host plant. Indeed, inoculation of plants with Tuber mycelia leads to an increased plant growth, indicating that the fungus is a mutualistic symbiont (Giovannetti and Fontana 1982). Recently, the genome sequence of a representative Tuber species, the black truffle T. melanosporum, has been determined (Martin et al. 2010). Although a large fraction of genes has been functionally defined by sequence homology, a consistent number of them $(>40 \%)$ remains functionally unknown. Reverse genetic analysis will thus be required in order to assign a role to these orphan genes. In Tuber, conventional genetic transformation procedures failed due to inefficient protoplast regeneration (Poma et al. 2006). We sought to circumvent this problem with the use of A. tumefaciensmediated transformation, a foreign DNA transfer strategy largely used in plants (Sheng and Citovsky 1996) and fungi (Michielse et al. 2005).

Our first attempt toward ATM-transformation in $T$. borchii was based on the pBGgHg binary vector (Grimaldi et al. 2005). Although the T-DNA entered host cells, transformation was transient and the organization of T-DNA (extrachromosomal vs. integrated) was not clear. Moreover, transformation efficiency was rather low $(0.2-0.5 \%)$ and the EGFP fluorescence signal as well as hygromycin resistance were lost quite early, typically after 4-5 weeks of in vitro culture. To overcome these limitations, while trying to achieve integrative transformation, we built two new binary vectors ( $\mathrm{pABr} 1$ and $\mathrm{pABr} 3)$. In both pABr1 and pABr3, GFP expression is driven by the strong ToxA promoter from the pathogenic fungus Phirenophora trittici repentis, which has been employed successfully for transgene overexpression in other filamentous fungi (Freitag et al. 2001; Lorang et al. 2001). Transformation with AGL-1/pABr1 (Figure 1a) produced stable transformants with a strong and long-lasting SGFP expression (Figure 1b). Also, transformation efficiency increased by about two-fold compared to AGL-1/pBGgHg (Figure 1c). This difference in transformation efficiency was confirmed with GV3101, an Agrobacterium strain less virulent than AGL-1 (Additional file 1: Figure S2).

A further increase in transformation efficiency ( 6fold compared to $\mathrm{pBGgHg}$ ) was obtained with $\mathrm{pABr} 3$ (Figure $3 \mathrm{a}, \mathrm{b}$ ), a shortened derivative of $\mathrm{pABr} 1$, lacking the unnecessary plant promoter/GFP/NOS terminator region (Figure 1a). This suggests that a smaller T-DNA size facilitates DNA transfer into host cells. Another important goal in order to strengthen selection efficiency was to maximize the concentration of hygromycin B that could be tolerated by transformed mycelia. Unlike transformation with the other AGL-1/binary vector combinations, AGL-1/pABr3 allowed the amplification of transformed mycelial clumps capable of growing in the presence of hygromycin B concentrations as high as $300 \mu \mathrm{g} / \mathrm{ml}$ (Figure 3c). This enabled the selective isolation and propagation of transformed mycelial regions. This is important, especially considering the lack of conidialike elements in Tuber spp., the syncytial nature of Tuber hyphae, and thus the fact that transformants are mosaics, likely bearing more than one transformed locus. We noted, however, that transformed hyphae are not randomly distributed within mycelia, but appear to be concentrated at the periphery of mycelial clumps, the only region from which the fungus could be propagated. This suggests that T-DNA may require a metabolically active tissue for activation and efficient functioning.

With pAbr3, the SGFP signal not only increased in intensity and distribution, but most of the analyzed mycelial sections appeared to be enriched in transformed hyphae even after 1 year from the initial transformation. In fact, in transformed mycelia, the GFP signal progressively became more diffuse and increasingly spread to contiguous hyphae (Figure 3a). This can be explained if the T-DNA is integrated in the Tuber genome, so that following duplication of a transformed nucleus the amount of produced/accumulated GFP gradually increases over time.

Integrative transfer was indeed demonstrated by PCR amplification of the ToxA (Figure 1d) and the $s g f p$ (Additional file 1: Figure S2d) sequences, as opposed to the negative amplification results obtained with the kanR gene region, which is located outside of the T-DNA (Figure 1d and Additional file 1: Figure S2d). This was further corroborated by the results of hybridization analyses conducted with region-specific probes. Most notably, the fact that the size of the fragment recognized by a probe hybridizing with the left T-DNA border (i.e., a region that is usually involved in the integration process (Thomas and Jones 2007)) increased from an expected size of 854 bp to $\sim 10,000 \mathrm{bp}$.

Lastly, we used transformed, hygromycin B-selected mycelia for the identification of the genomic sites of integration by TAIL-PCR (Liu and Whittier 1995), a widely used approach for the analysis of transformants in systems where homokaryotic colonies are hard to obtain (Poma et al. 2006). We sequenced T-DNA/Tuber genomic DNA flanking site amplicons obtained from transformed mycelia and found that two of them matched transposonrelated elements (Figure 4). In Tuber, repeated elements, including retrotransposons, account for about $60 \%$ of the genome (Martin et al. 2010), suggesting that integrative transformation mediated by heterologous recombination is likely driven by repetitive DNA sequences. 
The highly improved genetic transformation procedure described in this work represents a first, but crucial step toward a functional genomic analysis of Tuber, and perhaps other transformation-recalcitrant filamentous fungi as well, with the ultimate goal of producing a comprehensive site-specific mutant collection as in Aspergillus nidulans, N. crassa and Magnaporthe oryzae (http://www.fgsc.net/). Although whole genome sequence data are only available for $T$. melanosporum so far, we used T. borchii as an experimental organism to set up this improved transformation procedure because of the easier and more efficient propagation of its mycelium under axenic culture conditions. However, ongoing work, including the setting up of more efficient mycelial growth conditions, indicates that the optimized AGL1/pABr3-protocol here applied to T. borchii also works well in T. melanosporum, with the production of well detectable levels of GFP and resistance to hygromycin concentrations as high as $50 \mu \mathrm{g} / \mathrm{ml}$ (A.B and P.B., unpublished).

The next step will be the disruption of nonhomologous end joining (NHEJ) mediated by the Ku70 and Ku80 proteins in order to favor integration at homologous sites. The NHEJ pathway, discovered for the first time in mammals (Jeggo et al. 1991) and so named because it does not require homologous sequences to join DNA ends, repairs double-strand breaks in the absence of a homologous donor (Moore and Haber 1996). As shown in N. crassa (Ninomiya et al. 2004), disruption of the ku70/ku80 genes strongly enhances homologous recombination and thus the production of site-specific mutants. The presence of ku70/ku80 homologs in the T. melanosporum genome (GSTUMT00005220001 and GSTUMT000019 28001 gene models; http://mycor.nancy.inra.fr) suggests that such an approach may also be feasible in Tuber.

\section{Additional file}

Additional file 1: Figure S1. Outline of the ATM-transformation protocol; Figure S2. ATM-transformation with the pABr1 vector using GV3101 as recipient strain.

\section{Abbreviations \\ ATM: Agrobacterium tumefaciens-mediated; egfp: Enhanced green fluorescent protein; gpd: Glyceraldehyde-3-phosphate dehydrogenase; hph: Hygromycin B phosphotransferase; kanR: Kanamycin resistance gene, aminoglycoside 3'-phosphotransferase; LB: Left border; PDA: Potato-dextrose agar; RB: Right border; sgfp: Synthetic green fluorescent protein; TAIL-PCR: Thermal Asymmetric Interlaced PCR; T-DNA: Transfer DNA; Ti: Tumor-inducing.}

\section{Competing interests}

The authors declare that they have no competing interests.

\section{Authors' contributions}

$A B$ was in charge of plasmid vector construction and confocal microscopy analysis, set up the transformation protocol and prepared the first draft of the manuscript; BM contributed to plasmid vector construction, confocal microscopy analysis, final editing of the manuscript and figure assembly; EM performed Tail-PCR experiments; MP was in charge of integration site analyses and T. melanosporum experiments; PF contributed to data analysis; SO proposed the project, designed part of the experiments and wrote the last draft of the manuscript; PB coordinated the project, designed part of the experiments and contributed to manuscript drafting.

\section{Acknowledgments}

We are grateful to P. Romaine and to L. Ciuffetti for the kind gift of the $\mathrm{BGgHg}$ and $\mathrm{CT} 74$ plasmids, respectively. We thank R. Gerace for help in maintaining the Tuber strains, G. Buglia for skillful assistance with microscopy analysis and P. Costantino's group for the GV3101 A. tumefaciens strain. P.B. thanks La Sapienza University, Rome (Fondi di Ateneo) for partial financial support. A.B. was supported by a Teresa Ariaudo fellowship.

\section{Author details}

${ }^{1}$ Pasteur Cenci Bolognetti Foundation, c/o Department of Biology and Biotechnology "Charles Darwin", Sapienza University, Piazzale A. Moro 5, 00185 Rome, Italy. ${ }^{2}$ Department of Life Sciences, Biochemistry and Molecular Biology Unit, Laboratory of Functional Genomics and Protein Engineering, University of Parma, 43124 Parma, Italy. ${ }^{3}$ Institute of Biology and Molecular Pathology, CNR, 00185 Rome, Italy.

Received: 11 April 2014 Accepted: 27 April 2014

Published online: 29 May 2014

\section{References}

Ambra R, Grimaldi B, Zamboni S, Filetici P, Macino G, Ballario P (2004) Photomorphogenesis in the hypogeous fungus Tuber borchii: isolation and characterization of Tbwc-1, the homologue of the blue-light photoreceptor of Neurospora crassa. Fungal Genet Biol 41(7):688-697

Bundock P, Hooykaas PJ (1996) Integration of Agrobacterium tumefaciens T-DNA in the Saccharomyces cerevisiae genome by illegitimate recombination. Proc Natl Acad Sci USA 93(26):15272-15275

Bundock P, den Dulk-Ras A, Beijersbergen A, Hooykaas PJ (1995) Trans-kingdom T-DNA transfer from Agrobacterium tumefaciens to Saccharomyces cerevisiae. The EMBO journal 14(13):3206-3214

Bundock P, van Attikum H, den Dulk-Ras A, Hooykaas PJ (2002) Insertional mutagenesis in yeasts using T-DNA from Agrobacterium tumefaciens. Yeast 19 (6):529-536

Chen X, Stone M, Schlagnhaufer C, Romaine CP (2000) A fruiting body tissue method for efficient Agrobacterium-mediated transformation of Agaricus bisporus. Appl Environ Microbiol 66(10):4510-4513

Choi J, Park J, Jeon J, Chi MH, Goh J, Yoo SY, Park J, Jung K, Kim H, Park SY, Rho HS, Kim S, Kim BR, Han SS, Kang S, Lee YH (2007) Genome-wide analysis of T-DNA integration into the chromosomes of Magnaporthe oryzae. Mol Microbiol 66(2):371-382

Ciuffetti LM, Tuori RP, Gaventa JM (1997) A single gene encodes a selective toxin causal to the development of tan spot of wheat. Plant Cell 9(2):135-144

Freitag M, Ciuffetti LM, Selker EU (2001) Expression and visualization of Green Fluorescent Protein (GFP) in Neurospora crassa. Fungal Genet Newsletter 48:15-19

Giovannetti G, Fontana A (1982) Mycorrhizal synthesis between Cistaceae and Tuberaceae. New Phytol 92(4):533-537

Green MR, Sambrook J (2012) Molecular cloning: a laboratory manual, 4th edn. Laboratory Press, Cold Spring Harbor, NY

Grimaldi B, de Raaf MA, Filetici P, Ottonello S, Ballario P (2005) Agrobacteriummediated gene transfer and enhanced green fluorescent protein visualization in the mycorrhizal ascomycete Tuber borchii: a first step towards truffle genetics. Curr Genet 48(1):69-74

Hebe G, Hager A, Salzer P (1999) Initial signalling processes induced by elicitors of ectomycorrhiza-forming fungi in spruce cells can also be triggered by G-protein-activating mastoparan and protein phosphatase-inhibiting cantharidin. Planta 207(3):418-425

Jeggo PA, Tesmer J, Chen DJ (1991) Genetic analysis of ionising radiation sensitive mutants of cultured mammalian cell lines. Mutat Res 254(2):125-133

Lazo GR, Stein PA, Ludwig RA (1991) A DNA transformation-competent Arabidopsis genomic library in Agrobacterium. Biotechnology 9(10):963-967

Liu YG, Whittier RF (1995) Thermal asymmetric interlaced PCR: automatable amplification and sequencing of insert end fragments from P1 and YAC clones for chromosome walking. Genomics 25(3):674-681 
Lorang JM, Tuori RP, Martinez JP, Sawyer TL, Redman RS, Rollins JA, Wolpert TJ, Johnson KB, Rodriguez RJ, Dickman MB, Ciuffetti LM (2001) Green fluorescent protein is lighting up fungal biology. Appl Environ Microbiol 67(5):1987-1994

Martin F, Kohler A, Murat C, Balestrini R, Coutinho PM, Jaillon O, Montanini B, Morin E, Noel B, Percudani R, Porcel B, Rubini A, Amicucci A, Amselem J, Anthouard V, Arcioni S, Artiquenave F, Aury JM, Ballario P, Bolchi A, Brenna A, Brun A, Buee M, Cantarel B, Chevalier G, Couloux A, Da Silva C, Denoeud F, Duplessis S, Ghignone S et al (2010) Perigord black truffle genome uncovers evolutionary origins and mechanisms of symbiosis. Nature 464(7291):1033-1038

Mazars GR, Moyret C, Jeanteur P, Theillet CG (1991) Direct sequencing by thermal asymmetric PCR. Nucleic Acids Res 19(17):4783

Michielse CB, Hooykaas PJJ, van den Hondel CAMJJ, Ram AFJ (2005) Agrobacterium-mediated transformation as a tool for functional genomics in fungi. Curr Genet 48(1):1-17

Montanini B, Betti M, Marquez AJ, Balestrini R, Bonfante P, Ottonello S (2003) Distinctive properties and expression profiles of glutamine synthetase from a plant symbiotic fungus. Biochem J 373(Pt 2):357-368

Moore JK, Haber JE (1996) Cell cycle and genetic requirements of two pathways of nonhomologous end-joining repair of double-strand breaks in Saccharomyces cerevisiae. Mol Cell Biol 16(5):2164-2173

Ninomiya Y, Suzuki K, Ishii C, Inoue H (2004) Highly efficient gene replacements in Neurospora strains deficient for nonhomologous end-joining. Proc Natl Acad Sci U S A 101(33):12248-12253

Niwa Y (2003) A synthetic green fluorescent protein gene for plant biotechnology. Plant Biotechnology 20:1-11

Pegler DN, Spooner BM, Young TWK (1993) British truffles: a revision of British hypogeous fungi. Royal Botanic Gardens, Kew, London, UK

Poma A, Limongi T, Pacioni G (2006) Current state and perspectives of truffle genetics and sustainable biotechnology. Appl Microbiol Biotechnol 72(3):437-441

Rolland S, Jobic C, Fevre M, Bruel C (2003) Agrobacterium-mediated transformation of Botrytis cinerea, simple purification of monokaryotic transformants and rapid conidia-based identification of the transfer-DNA host genomic DNA flanking sequences. Curr Genet 44(3):164-171

Sheng J, Citovsky V (1996) Agrobacterium-plant cell DNA transport: have virulence proteins, will travel. Plant Cell 8(10):1699-1710

Singer K, Shiboleth YM, Li J, Tzfira T (2012) Formation of complex extrachromosomal T-DNA structures in Agrobacterium tumefaciens-infected plants. Plant Physiol 160(1):511-522

Thomas CM, Jones JD (2007) Molecular analysis of Agrobacterium T-DNA integration in tomato reveals a role for left border sequence homology in most integration events. Mol Genet Genomics 278(4):411-420

Wang JY, Li HY (2008) Agrobacterium tumefaciens-mediated genetic transformation of the phytopathogenic fungus Penicillium digitatum. J Zhejiang Univ Sci 9(10):823-828

Zupan J, Muth TR, Draper O, Zambryski P (2000) The transfer of DNA from Agrobacterium tumefaciens into plants: a feast of fundamental insights. Plant J 23(1):11-28

doi:10.1186/s13568-014-0043-x

Cite this article as: Brenna et al.: Integrative gene transfer in the truffle Tuber borchii by Agrobacterium tumefaciens-mediated transformation. AMB Express 2014 4:43.

\section{Submit your manuscript to a SpringerOpen ${ }^{\circ}$ journal and benefit from:}

- Convenient online submission

- Rigorous peer review

- Immediate publication on acceptance

- Open access: articles freely available online

- High visibility within the field

- Retaining the copyright to your article

Submit your next manuscript at $\gg$ springeropen.com 